\title{
Space-Time Network Codes Utilizing Transform-Based Coding
}

\author{
Hung-Quoc Lai ${ }^{1,2}$, Zhenzhen $\mathrm{Gao}^{2,3}$, and K. J. Ray $\mathrm{Liu}^{2}$ \\ ${ }^{1}$ US Army RDECOM CERDEC, RDER-STW-N, Aberdeen Proving Ground, MD 21005, USA. \\ ${ }^{2}$ Department of Electrical and Computer Engineering,University of Maryland, College Park, MD 20742, USA. \\ ${ }^{3}$ Department of Information and Communication Engineering, Xian Jiaotong University, Xi'an, P. R. China 710049.
}

\begin{abstract}
Cooperative communications can be used to improve communication reliability. However, the transmissions from different relaying nodes result in a great challenge in practice. The use of TDMA for relaying transmissions causes large transmission delay. In contrast, FDMA and CDMA associate with the issue of imperfect frequency and timing synchronization due to the simultaneous transmissions from the relaying nodes. In this work, we propose a space-time network coding (STNC) scheme utilizing transform-based coding to improve communication reliability while maintaining a stable network throughput and overcoming the issue of imperfect frequency and timing synchronization. Based on TDMA, a node in the network forms a linearly-coded signal from the overheard symbols and transmits it to the destination in its dedicated time slot. The pairwise error probability (PEP) is analyzed and design criteria of the STNC are derived to ensure achieving full diversity. Simulations are conducted to verify the performance of the proposed scheme and to reveal its advantage over a distributed space-time block coding scheme under timing synchronization errors.
\end{abstract}

\section{INTRODUCTION}

It is well-known that performance of communication systems degrades greatly when operating in radio frequency environments characterized by multipath propagation such as urban environments. Diversity techniques such as time diversity, frequency diversity, and spatial diversity can improve transmission reliability. Among these techniques, spatial diversity achieved by cooperative communications [1] has become recently attractive.

In cooperative communications, nodes acting as relays retransmit the overheard information to a destination. The distributed antennas among nodes are used to provide spatial diversity without the need to use multiple antennas at the source. Various cooperative diversity protocols have been proposed and analyzed in [2], [3], [4]. They often consist of two phases: source transmission and relay transmission. In the first phase, a source broadcasts its information to a destination and the relays, which then forward the overheard information to the destination in the second phase.

Much of research in cooperative communications has focused on simultaneous transmission from two or more nodes with an assumption of perfect frequency and timing synchronization [4], [5]. However, such an assumption is difficult to be met in practice, especially in mobile conditions where nodes move at different speeds and in different directions. The imperfect synchronization causes the intersymbol interference (ISI), which is the source of system performance degradation [6], [7]. For timing synchronization, the coordination to make signals received simultaneously at the destination is challenging due to differences in propagation time among nodes, processing time in each radio, and timing estimation error. The frequency synchronization issue occurs when each node has an independent local oscillator generating a transmit frequency with certain variation to the nominal. Various frequency mismatches occur at once at the destination and make it difficult to estimate and compensate all the frequency offsets.

To overcome the imperfect synchronization issue that prohibits two or more nodes from transmitting at the same time, time-division multiple access (TDMA) would be the most commonly-used technique in many applications. However, TDMA is extremely inefficient in cooperative communications, especially for large numbers of relay nodes to achieve high diversity orders. For a network of $N$ client nodes helping one another in transmitting their information to a common base node, TDMA requires $N^{2}$ time slots [4], causing large transmission delays as $N$ increases. Therefore, there is an essential need to reduce the total required time slots in TDMA for cooperative communications while maintaining the spatial diversity and overcoming the issue of imperfect frequency and timing synchronization.

The recent work on space-time network coding (STNC) [8] reveals the mechanism to achieve the foretold objectives. A client node acting as a relay linearly combines the set of symbols as a unique signal and transmits it to the base node in its dedicated time slot. As a result, the foretold issue of imperfect synchronization disappears and only $2 N$ time slots are required, a significant reduction from that in traditional cooperative communications using TDMA.

The combining technique in this work is different from that in [8], where symbols at a client node are formed in CDMA or OFDMA manners. In this work, transform-based coding [9], [10], whose coding matrices compose a set of parameters that are optimized for conventional signal constellations, is used to generate the code symbols. The combining of symbols at a client node here is also different from that in traditional network coding [11]. Such a combining technique could be possible and subject to a future investigation. We analyze the pairwise error probability (PEP) performance of the proposed STNC scheme and derive the design criteria of the network coding matrix. Simulations are provided to verify the proposed STNC achieves full diversity. In addition, a performance comparison between the proposed STNC and a distributed space-time block coding (DSTBC) scheme under timing synchronization errors is investigated.

The rest of this paper is organized as follows. After this introduction section, STNC utilizing transform-based coding is introduced in Section II. The system model and signal detection are also presented in this section. The performance analysis and the code design is derived in Section III. In Section IV, the impact of timing synchronization errors on DSTBC performance is presented. The simulations and conclusion are provided in Section V and Section VI, respectively.

Notation: Lower and upper case bold symbols denote column vectors and matrices, respectively. ${ }^{*},{ }^{\mathcal{T}}$, and $\mathcal{H}$ de- 


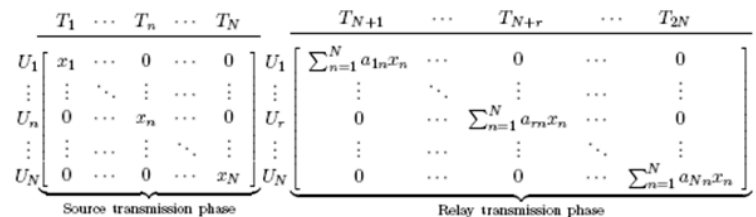

Fig. 1. Space-time network codes utilizing transform-based coding.

note complex conjugate, transpose, and Hermitian transpose, respectively. $\mathrm{E}\{\},. \operatorname{diag}\{$.$\} , and |$.$| represent the expectation,$ a diagonal matrix, the size of a set, respectively.

\section{System Model and Signal Detection}

We consider a wireless network that consists of $(N+1)$ nodes denoted as $U_{0}, U_{1}, U_{2}, \ldots, U_{N}$, where $U_{1}, U_{2}, \ldots, U_{N}$ are source nodes transmitting their information to the common destination node $U_{0}$. For convenience, we refer $U_{0}$ as a base node and $U_{1}, U_{2}, \ldots, U_{N}$ as client nodes as in cellular networks. In this work, we consider the base node has $M$ antennas while the client nodes are single-antenna devices. The channels are modelled as narrow-band Rayleigh fading with additive white Gaussian noise (AWGN). We assume that the antenna separations are at least a half of wavelengths apart and thus the channels are spatially uncorrelated. The channel coefficient between an arbitrary receiver $u$ and transmitter $v$ is defined as $h_{u v} \sim \mathcal{C N}\left(0, \sigma_{u v}^{2}\right)$, where $\sigma_{u v}^{2}$ is the channel variance.

\section{A. System Model}

The system model comprises a source transmission phase and a relay transmission phase. Each transmission phase consists of $N$ time slots, and the network requires $2 N$ time slots for transmissions of the $N$ symbols to $U_{0}$. In the source transmission phase, each client node $U_{n}$ for $n=1,2, \ldots, N$ is assigned a time slot, denoted $T_{n}$, to broadcast its symbol $x_{n}$ to other client nodes $U_{r}, r \neq n$, where $x_{n}$ is from an $\mathcal{M}$-QAM constellation $\mathbb{X}$. At the end of this phase, each client node $U_{r}$ for $r=1,2, \ldots, N$ possesses a set of $N$ symbols $x_{1}, x_{2}, \ldots, x_{N}$, comprising its own symbol $x_{r}$. In the relay transmission phase, $U_{r}$ forms a single linearly-coded signal, a linear combination of the overheard symbols and its own one, and transmits the signal to the base node in its dedicated time slot $T_{N+r}$. $U_{r}$ detects the symbol $x_{n}$ based on the source signal and reencode the symbol in its linearly-coded signal if the decoding is successful, the so called DF protocol. A detection state, a success or a failure in detecting a symbol, can be determined based on the amplitude of the estimated channel coefficient [2] or the received signal-to-noise ratio (SNR) [4]. Notice that this DF scheme is also called the selective-relaying protocol in literature [2]. The transmissions from the $N$ client nodes in the source and relay transmission phases are illustrated in Figure 1, which guarantee a single transmission in the network at any given time slot to eliminate the issue of imperfect synchronization in traditional cooperative communications.

The received signal at $U_{r}$ from $U_{n}$ in the source transmission phase is

$$
y_{r n}=h_{r n} \sqrt{P_{n}} x_{n}+w_{r n}
$$

where $P_{n}$ is the transmit power at $U_{n}$ in the source transmission phase and $w_{r n} \sim \mathcal{C N}\left(0, N_{0}\right)$ is the AWGN. In the relay transmission phase, the received signal at the $m$ th antenna, $m=1,2, \ldots, M$, of the base node $U_{0}$ from $U_{r}$ is

$$
y_{m r}=h_{m r} \sqrt{P_{r}} s_{r}+w_{m r}
$$

where $P_{r}$ is the transmit power in the relay transmission phase, $w_{m r} \sim \mathcal{C N}\left(0, N_{0}\right)$ is the AWGN, and

$$
s_{r}=\sum_{n=1}^{N} a_{r n} \beta_{r n} x_{n}=\mathbf{a}_{r}^{\mathcal{T}} \mathbf{B}_{r} \mathbf{x}
$$

is the linearly-coded symbol at $U_{r}$. In (3), $\mathbf{a}_{r}=\left[a_{r 1}, \ldots, a_{r n}\right.$, $\left.\ldots, a_{r N}\right]^{\mathcal{T}}, \mathbf{a}_{r}^{\mathcal{T}} \mathbf{a}_{r}=1$ to normalize the transmitted signal, is the code vector at $U_{r}$ with $a_{r n}$ being the code coefficient associated with $x_{n}, \mathbf{x}=\left[x_{1}, x_{2}, \ldots, x_{N}\right]^{\mathcal{T}}$ is the transmitted symbol vector, and $\mathbf{B}_{r}=\operatorname{diag}\left\{\beta_{r 1}, \ldots, \beta_{r n}, \ldots, \beta_{r N}\right\}$ is a matrix representing the detection state at $U_{r}$ with $\beta_{r n}$ being detection state associated with $x_{n}$. We have $\beta_{r r}=1$ always since $U_{r}$ has its own symbol $x_{r}$ and for $r \neq n$,

$$
\beta_{r n}=\left\{\begin{array}{ll}
1 & \text { if } U_{r} \text { decodes } x_{n} \text { correctly } \\
0 & \text { otherwise }
\end{array} .\right.
$$

The received signals at $U_{0}$ can be expressed in a matrix form as

$$
\mathbf{Y}=\mathbf{H S}+\mathbf{W},
$$

where $\mathbf{Y}, \mathbf{H}$, and $\mathbf{W}$ are $M \times N$ matrices comprising the received signals, the channel coefficients, and the AWGN's, and $\mathbf{S}=\operatorname{diag}\left\{\sqrt{P_{1}} s_{1}, \ldots, \sqrt{P_{r}} s_{r}, \ldots, \sqrt{P_{N}} s_{N}\right\}$ is the $N \times N$ code matrix.

\section{B. Signal Detection}

To detect the transmitted symbols, we assume that receivers have a full knowledge of the channel state information (CSI), which can be acquired using a preamble in the transmitted signal as usually done in systems such as 802.11 [12]. We also assume that the base node knows the detection states at the relay nodes. This can be done by using an $N$-bit indicator in the relaying signal. Notice that in practice, information is transmitted in packets [12] that contain a large number of symbols. Each packet is detected as a whole, and a CRC [13] is sufficient to determine the detection state of the packet. Thus one bit per packet results in a minimal overhead.

To derive code criteria in the next section, maximumlikelihood (ML) detectors, which is a minimum distance rule, are used. The detected symbol vector at the base node $U_{0}$ is

$$
\widehat{\mathbf{x}}=\underset{\mathbf{x} \in \mathbb{X}^{N}}{\operatorname{argmin}}\left\{\|\mathbf{Y}-\mathbf{H S}\|_{\mathcal{F}}^{2}\right\},
$$

where $\|\cdot\|_{\mathcal{F}}$ denotes the Frobenius norm. As shown in (6), detecting $\mathrm{x}$ requires testing $|\mathbb{X}|^{N}$ possible vectors $\mathrm{x}$, which is computationally prohibitive as $N$ and/or $|\mathbb{X}|$ are large. In that case, sphere decoder [14], which results in suboptimal decoding, can be used. The detection of $x_{n}$ at client node $U_{r}$ in the source transmission phase follows

$$
\widehat{x}_{n}=\underset{x \in \mathbb{X}}{\operatorname{argmin}}\left\{\left|y_{r n}-h_{r n} \sqrt{P_{n n}} x\right|^{2}\right\} .
$$

\section{Performance Analysis and Code Design}

Let us define $\mathbf{y}=\operatorname{vec}(\mathbf{Y}), \mathbf{h}=\operatorname{vec}(\mathbf{H})$, and $\mathbf{w}=\operatorname{vec}(\mathbf{W})$, where $\operatorname{vec}($.$) denotes the vectorization of a matrix by stacking$ the columns of the matrix on the top of one another. Then the signal matrix in (5) can be rewritten in vector form as

$$
\mathbf{y}=\mathbf{D h}+\mathbf{w},
$$


where $\mathbf{D}=\mathbf{S} \otimes \mathbf{I}_{M}$ with $\otimes$ denoting the Kronecker product [15] and $\mathbf{I}_{M}$ being the identity matrix of size $M$. Suppose that $\mathbf{D}$ and $\widetilde{\mathbf{D}}$ are two different matrices related to two different code matrices $\mathbf{S}$ and $\widetilde{\mathbf{S}}$. The base node applies the ML detector in (6), and thus the conditional PEP is given by

$$
\begin{array}{r}
\operatorname{Prob}\left(\mathbf{S} \rightarrow \widetilde{\mathbf{S}} \mid\left\{\mathbf{B}_{r}\right\}, \mathbf{h}\right)=\operatorname{Prob}\left(\|\mathbf{y}-\mathbf{D h}\|^{2}>\right. \\
\left.\|\mathbf{y}-\widetilde{\mathbf{D}} \mathbf{h}\|^{2} \mid\left\{\mathbf{B}_{r}\right\}, \mathbf{h}, \mathbf{S} \text { transmitted }\right) .
\end{array}
$$

The conditional PEP can be expressed in a quadratic form of a complex Gaussian random variable as

$$
\operatorname{Prob}\left(\mathbf{S} \rightarrow \widetilde{\mathbf{S}} \mid\left\{\mathbf{B}_{r}\right\}, \mathbf{h}\right)=\operatorname{Prob}\left(Q<0 \mid\left\{\mathbf{B}_{r}\right\}, \mathbf{h}\right),
$$

where

$$
Q=\left[\begin{array}{ll}
\mathbf{z}_{1}^{\mathcal{H}} & \mathbf{z}_{2}^{\mathcal{H}}
\end{array}\right]\left[\begin{array}{cc}
\mathbf{I}_{M N} & \mathbf{0} \\
\mathbf{0} & -\mathbf{I}_{M N}
\end{array}\right]\left[\begin{array}{l}
\mathbf{z}_{1} \\
\mathbf{z}_{2}
\end{array}\right],
$$

in which $\mathbf{0}$ represents a zero matrix, $\mathbf{z}_{1}=(\mathbf{D}-\widetilde{\mathbf{D}}) \mathbf{h}+\mathbf{w}$, and $\mathbf{z}_{2}=\mathbf{w}$. Because $\mathbf{h}$ and $\mathbf{w}$ are mutually independent random vectors, the conditional PEP can be averaged over the channel realization $\mathbf{h}$. At high SNR, the conditional PEP between $\mathbf{S}$ and $\widetilde{\mathbf{S}}$ can be upper-bounded as [16]

$$
\operatorname{Prob}\left(\mathbf{S} \rightarrow \widetilde{\mathbf{S}} \mid\left\{\mathbf{B}_{r}\right\}\right) \leq\left(\begin{array}{c}
2 \nu-1 \\
\nu-1
\end{array}\right) N_{0}^{\nu} \prod_{i=1}^{\nu} \gamma_{i}^{-1},
$$

where $\nu$ and $\gamma_{i}$ is the rank and the $i$ th non-zero eigenvalue of the matrix $(\mathbf{D}-\widetilde{\mathbf{D}}) \mathbf{R}_{\mathbf{h}}(\mathbf{D}-\widetilde{\mathbf{D}})^{\mathcal{H}}$ with $\mathbf{R}_{\mathbf{h}} \triangleq \mathrm{E}\left\{\mathbf{h h}^{\mathcal{H}}\right\}=$ $\operatorname{diag}\left\{\sigma_{11}^{2}, \ldots, \sigma_{M 1}^{2}, \sigma_{12}^{2}, \ldots, \sigma_{M 2}^{2}, \ldots, \sigma_{1 N}^{2}, \ldots, \sigma_{M N}^{2}\right\}$ being the correlation matrix of the channel vector $\mathbf{h}$. Based on the relationship between $\mathbf{D}$ and $\mathbf{S}$ in (8) and the fact that $\mathbf{D}, \widetilde{\mathbf{D}}$, and $\mathbf{R}_{\mathbf{h}}$ are all diagonal matrices, [15]

$$
(\mathbf{D}-\widetilde{\mathbf{D}}) \mathbf{R}_{\mathbf{h}}(\mathbf{D}-\widetilde{\mathbf{D}})^{\mathcal{H}}=\left(\boldsymbol{\Delta}_{\mathbf{S}} \otimes \mathbf{I}_{M}\right) \mathbf{R}_{\mathbf{h}}
$$

where we define

$$
\begin{aligned}
\boldsymbol{\Delta}_{\mathbf{S}} \triangleq & (\mathbf{S}-\widetilde{\mathbf{S}})(\mathbf{S}-\widetilde{\mathbf{S}})^{\mathcal{H}} \\
= & \operatorname{diag}\left\{P_{1}\left|\mathbf{a}_{1}^{\mathcal{T}} \mathbf{B}_{1} \Delta \mathbf{x}\right|^{2}, \ldots, P_{r}\left|\mathbf{a}_{r}^{\mathcal{T}} \mathbf{B}_{r} \Delta \mathbf{x}\right|^{2},\right. \\
& \left.\ldots, P_{N}\left|\mathbf{a}_{N}^{\mathcal{T}} \mathbf{B}_{N} \Delta \mathbf{x}\right|^{2}\right\},
\end{aligned}
$$

in which $\Delta \mathrm{x}=\mathrm{x}-\widetilde{\mathrm{x}}$ with two distinct transmitted symbol vectors $\mathbf{x}$ and $\widetilde{\mathbf{x}}$. Given that $\operatorname{rank}\left(\mathbf{R}_{\mathbf{h}}\right)=M N, \nu=M \nu_{\mathbf{S}}$, where $\nu_{\mathbf{S}} \triangleq \operatorname{rank}\left(\boldsymbol{\Delta}_{\mathbf{S}}\right)$. In (14), the maximum rank of $\boldsymbol{\Delta}_{\mathbf{S}}$ is $N$. We should design the coding vectors $\mathbf{a}_{r}$ 's to achieve this rank and the design criteria will be derived later in the section. In that case, the $N$ eigenvalues of $\boldsymbol{\Delta}_{\mathbf{S}}$ are $\lambda_{r}=P_{r}\left|\mathbf{a}_{r}^{\mathcal{T}} \mathbf{B}_{r} \Delta \mathbf{x}\right|^{2}$ for $r=1,2, \ldots, N$, and we can express (12) as

$$
\begin{array}{r}
\operatorname{Prob}\left(\mathbf{S} \rightarrow \widetilde{\mathbf{S}} \mid\left\{\mathbf{B}_{r}\right\}\right) \leq\left(\begin{array}{c}
2 M N-1 \\
M N-1
\end{array}\right) N_{0}^{M N} \times \\
\left(\prod_{m=1}^{M} \prod_{r=1}^{N} \frac{1}{\sigma_{m r}^{2}}\right)\left(\prod_{r=1}^{N}\left(P_{r}\left|\mathbf{a}_{r}^{\mathcal{T}} \mathbf{B}_{r} \Delta \mathbf{x}\right|^{2}\right)^{-M}\right) .
\end{array}
$$

Averaging with respect to the detection state matrices $\left\{\mathbf{B}_{r}\right\}$ with a notice that the detection state matrices are mutually independent because the detection at each client node is independent from one another, the PEP can be rewritten as

$$
\begin{aligned}
\operatorname{Prob}(\mathbf{S} \rightarrow \widetilde{\mathbf{S}}) & \leq\left(\begin{array}{c}
2 M N-1 \\
M N-1
\end{array}\right) N_{0}^{M N}\left(\prod_{m=1}^{M} \prod_{r=1}^{N} \frac{1}{\sigma_{m r}^{2}}\right) \\
& \times \underbrace{\prod_{r=1}^{N} \mathrm{E}\left\{\left(P_{r}\left|\sum_{n=1}^{N} a_{r n} \beta_{r n} \Delta x_{n}\right|^{2}\right)^{-M}\right\}}_{\mathcal{A}},
\end{aligned}
$$

where $\Delta x_{n}=x_{n}-\widetilde{x}_{n}$.

Let $S_{r} \triangleq\left[\beta_{r 1}, \ldots, \beta_{r n}, \ldots, \beta_{r N}\right]_{2}$ for $n \neq r$, where $[.]_{2}$ denotes a base-2 number. $S_{r}$ is a decimal number representing one of $2^{N-1}$ detection states at $U_{r}$. Because symbols at a client node are independently detected, $\beta_{r n}$ 's are independent Bernoulli random variables with a distribution

$$
G\left(\beta_{r n}\right)=\left\{\begin{array}{cc}
1-p_{r n} & \text { if } \beta_{r n}=1 \\
p_{r n} & \text { if } \beta_{r n}=0
\end{array},\right.
$$

where $p_{r n}$ is the symbol error rate (SER) for detecting $x_{n}$ at $U_{r}$. For $\mathcal{M}$-QAM modulation, it can be shown that [4]

$$
p_{r n}=F\left(1+\frac{b \sigma_{r n}^{2} P_{n}}{N_{0} \sin ^{2}(\theta)}\right),
$$

where $b=\frac{3}{2(\mathcal{M}-1)}$ and

$$
F(g(\theta))=\frac{4 C}{\pi} \int_{0}^{\frac{\pi}{2}} \frac{1}{g(\theta)} d \theta-\frac{4 C^{2}}{\pi} \int_{0}^{\frac{\pi}{4}} \frac{1}{g(\theta)} d \theta,
$$

in which $C=1-\frac{1}{\sqrt{\mathcal{M}}}$ and $g(\theta)$ denotes a function of $\theta$. At high SNR, we can ignore the 1 in (18) and obtain

$$
p_{r n} \simeq \mathcal{K} \frac{N_{0}}{\sigma_{r n}^{2} P_{n}},
$$

where

$$
\mathcal{K}=\frac{4 C}{b \pi} \int_{0}^{\frac{\pi}{2}} \sin ^{2} \theta d \theta-\frac{4 C^{2}}{b \pi} \int_{0}^{\frac{\pi}{4}} \sin ^{2} \theta d \theta .
$$

Hence the probability of detection state at $U_{r}$ in state $S_{r}$ is

$$
\operatorname{Prob}\left(S_{r}\right)=\prod_{n=1 ; n \neq r}^{N} G\left(\beta_{r n}\right) \simeq \prod_{n=1 ; \beta_{r n}=0}^{N} p_{r n},
$$

where we use the approximation $1-p_{r n} \simeq 1$ for high SNR.

Given a detection state $S_{r}$, which can take $2^{N-1}$ values,

$\mathcal{A} \simeq \prod_{r=1}^{N} \sum_{S_{r}=0}^{2^{N-1}-1}\left(\frac{1}{P_{r}\left|\sum_{n=1 ; \beta_{r n}=1}^{N} a_{r n} \Delta x_{n}\right|^{2}}\right)^{M} \prod_{n=1 ; \beta_{r n}=0}^{N} p_{r n}$.

Substituting (20) into (23) and ignoring high order terms of SERs, we can show that

$$
\mathcal{A} \simeq P^{-M N} \prod_{r=1}^{N}\left(\frac{1}{\left|\mathbf{a}_{r}^{\mathcal{T}} \mathbf{\Delta} \mathbf{x}\right|^{2}}\right)^{M}\left(\frac{1}{\alpha_{r}^{M}}+\sum_{n=1 ; n \neq r}^{N} \frac{\mathcal{K} N_{0}\left(d_{\max }^{2}\right)^{M}}{\alpha_{n} \sigma_{r n}^{2}}\right),
$$

where $d_{\max }$ is the maximum Euclidean distance of $\mathbb{X}$ and $\alpha_{r}=P_{r} / P$ and $\alpha_{n}=P_{n} / P$ are the fractions of total transmit power $P$ allocated for the source and relay transmission phases, respectively. Substituting (24) into (16), the PEP is

$$
\begin{aligned}
& \operatorname{Prob}(\mathbf{S}\rightarrow \widetilde{\mathbf{S}}) \leq\left(\begin{array}{c}
2 M N-1 \\
M N-1
\end{array}\right) \prod_{m=1}^{M} \prod_{r=1}^{N}\left(\frac{1}{\sigma_{m r}^{2}\left|\mathbf{a}_{r}^{\mathcal{I}} \mathbf{\Delta} \mathbf{x}\right|^{2}}\right. \\
&\left.\times\left(\frac{1}{\alpha_{r}^{M}}+\sum_{n=1 ; n \neq r}^{N} \frac{\mathcal{K} N_{0}\left(d_{\max }^{2}\right)^{M}}{\alpha_{n} \sigma_{r n}^{2}}\right)\right)\left(\frac{P}{N_{0}}\right)^{-M N} .
\end{aligned}
$$




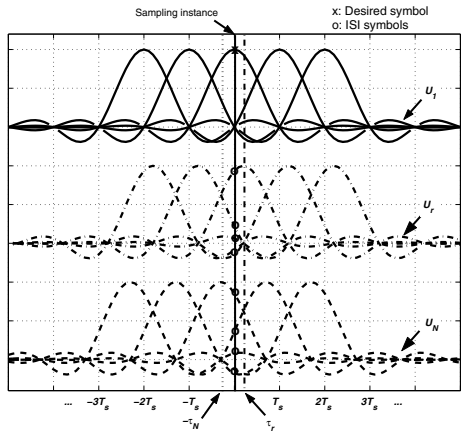

Fig. 2. Impact of timing synchronization errors on DSTBC.

From (25), the design criteria for the coding vectors $\mathbf{a}_{r}$ 's are as follows.

Diversity criterion: The system provides full diversity with order $M N$ if $\left|\mathbf{a}_{r}^{\mathcal{T}} \boldsymbol{\Delta} \mathbf{x}\right| \neq 0, \forall r \in[1, N], \forall \mathbf{x}, \widetilde{\mathbf{x}} \in \mathbb{X}$.

Product criterion: The minimum value of the product $\prod_{r=1}^{N} \mathbf{a}_{r}^{\mathcal{T}} \Delta \mathbf{x}$ over all pairs of distinct symbol vectors $\mathbf{x}$ and $\widetilde{\mathbf{x}}$ should be as large as possible. The product criterion is of secondary importance and should be optimized if full diversity is achieved.

Let $\mathbf{A} \triangleq\left[\mathbf{a}_{1}, \ldots, \mathbf{a}_{r}, \ldots, \mathbf{a}_{N}\right]$ be the coding matrix. With the above design criteria, $\mathbf{A}$ has been proposed in a number of previous work [9], [10], [17], where Hadamard and Vandermonde matrices were used to construct A. Note that the coding matrices based on Vandermonde matrices result in larger minimum product values than those using Hadamard matrices. Thus we will use Vandermonde matrices for our STNC scheme. In that case,

$$
\mathbf{A}=\frac{1}{\sqrt{N}}\left[\begin{array}{ccccc}
1 & \cdots & 1 & \cdots & 1 \\
\theta_{1} & \cdots & \theta_{r} & \cdots & \theta_{N} \\
\vdots & \vdots & \vdots & \vdots & \vdots \\
\theta_{1}^{N-1} & \cdots & \theta_{r}^{N-1} & \cdots & \theta_{N}^{N-1}
\end{array}\right],
$$

where $\theta_{r}$ depends on $N$ and the construction methods [9], [10], [17].

\section{IMPACT OF SYNCHRONIZATION ERRORS ON DSTBC}

The source transmission phase of schemes using DSTBC is the same with that of STNC scheme, i.e., that the $N$ client nodes take turn to exchange their transmit symbols among themselves. Thus the signal model for this phase follows (1). After the source transmission phase, a STBC matrix is formed from the overheard symbol vector $\mathbf{x}$ as

$$
\mathbf{S}(l)=\left[\begin{array}{ccccc}
s_{01}(l) & \cdots & s_{0 r}(l) & \cdots & s_{0 N}(l) \\
\vdots & \ddots & \vdots & \cdots & \vdots \\
s_{k 1}(l) & \cdots & s_{k r}(l) & \cdots & s_{k N}(l) \\
\vdots & \cdots & \vdots & \ddots & \vdots \\
s_{(K-1) 1}(l) & \cdots & s_{(K-1) r}(l) & \cdots & s_{(K-1) N}(l)
\end{array}\right],
$$

where $l$ denotes the code matrix at frame $l$, which consists of $K$ time slots required to transmit the code matrix, and $s_{k r}(l)$ is the code symbol transmitted at time slot $k$ and through client node $U_{r}$. For example, the code matrix for two cooperative client nodes is the well-known Alamouti STBC [18]

$$
\left[\begin{array}{cc}
x_{1}(l) & x_{2}(l) \\
-x_{2}^{*}(l) & x_{1}^{*}(l)
\end{array}\right]
$$

Notice that when a symbol is erroneously decoded at a client node, the associated code symbols at that node are set to zero and the node remains silent during that time slot [5].
In the relay transmission phase, the symbols in the $k$ th row of the code matrix (27) for $k=0,1, \ldots, K-1$ are simultaneously transmitted through the $N$ client nodes, each acting as a respective antenna of a traditional MIMO system. Due to the time synchronization errors, the received signal at antenna $m$ and time slot $k$ of frame $l$ is

$$
\begin{gathered}
y_{m}^{k}(l)=\sqrt{\frac{P_{r}}{K}} \sum_{r=1}^{N} h_{m r}(l) \sum_{q=-q_{0}}^{q_{0}} s_{(\bmod (k+q, K)) r} \\
\left(l+\left\lfloor\frac{k+q}{K}\right\rfloor\right) p\left((q-l) T_{s}-\tau_{r}\right)+w_{m k}(l),
\end{gathered}
$$

where mod and $\lfloor$.$\rfloor denote the modulo and floor operations,$ respectively, $w_{m k}(l) \sim \mathcal{C N}\left(0, N_{0}\right)$ is the AWGN,

$$
p(t)=\frac{\sin \left(\pi t / T_{s}\right)}{\pi t / T_{s}} \frac{\cos \left(\pi \beta t T_{s}\right)}{1-4 \beta^{2} t^{2} T_{s}^{2}}
$$

is the raised cosine pulse shape with a symbol period $T_{s}$ and the roll-off factor $\beta$ [19], $q_{0}$ is the number of nearest neighbor symbols from each side (left or right) that cause the ISI on the symbol of interest, and $\tau_{r}$ is the timing error associated with the signal from $U_{r}$. Figure 2 illustrates the ISI effect on the desired symbol.

Because the base node assumes perfect timing synchronization, it applies the detection techniques in [20]. For example in the case of $N=2$,

$$
\begin{aligned}
\widehat{x}_{1}(l)= & \underset{x_{1} \in|\mathbb{X}|}{\operatorname{argmin}}\left\{\mid\left[\sum _ { m = 1 } ^ { M } \left(y_{m}^{0}(l) \sqrt{\frac{P_{1}}{2}} h_{m 1}^{*}(l)\right.\right.\right. \\
& \left.\left.+\left(y_{m}^{1}(l)\right)^{*} \sqrt{\frac{P_{2}}{2}} h_{m 2}(l)\right)\right]-\left.x_{1}\right|^{2} \\
& \left.+\left(-1+\sum_{m=1}^{M} \sum_{r=1}^{2} \frac{P_{r}}{2}\left|h_{m r}(l)\right|^{2}\right)\left|x_{1}\right|^{2}\right\} \\
\widehat{x}_{2}(l)= & \underset{x_{2} \in|\mathbb{X}|}{\operatorname{argmin}}\left\{\mid\left[\sum _ { m = 1 } ^ { M } \left(y_{m}^{0}(l) \sqrt{\frac{P_{2}}{2}} h_{m 2}^{*}(l)\right.\right.\right. \\
& \left.\left.-\left(y_{m}^{1}(l)\right)^{*} \sqrt{\frac{P_{1}}{2}} h_{m 1}(l)\right)\right]-\left.x_{2}\right|^{2} \\
& \left.+\left(-1+\sum_{m=1}^{M} \sum_{r=1}^{2} \frac{P_{r}}{2}\left|h_{m r}(l)\right|^{2}\right)\left|x_{2}\right|^{2}\right\} .
\end{aligned}
$$

This detection inherits the ISI effect in (29) and thus degrades the performance as we will see in the next section.

\section{Simulations}

Here we perform computer simulations to verify our proposed STNC scheme and to compare the performance of our scheme with that of those utilizing DSTBC, with and without timing synchronization errors. QPSK modulation is used for the transmitted symbols. We assume that the $N$ client nodes are in a cluster with the same channel variances $\sigma_{r n}^{2}=30$ among them. The cluster is faraway from the base node and thus the channel variance between the base node and a client node $\sigma_{0 n}^{2}=1$ is the same for all client nodes. Also we assume the noise variance $N_{0}=1$. Equal power allocation is assumed, where a total transmit power $P$ associated with transmitted symbol is divided equally among the transmissions. In this case, $P_{n}=P /(N+1)$ and $P_{r}=P N /(N+1)$ for STNC scheme and $P_{n}=P /(K+1)$ and $P_{r}=P K /(K+1)$ for DSTBC scheme required $K$ time slots for transmitting the code matrix. The total transmit power $P$ is the same with that 


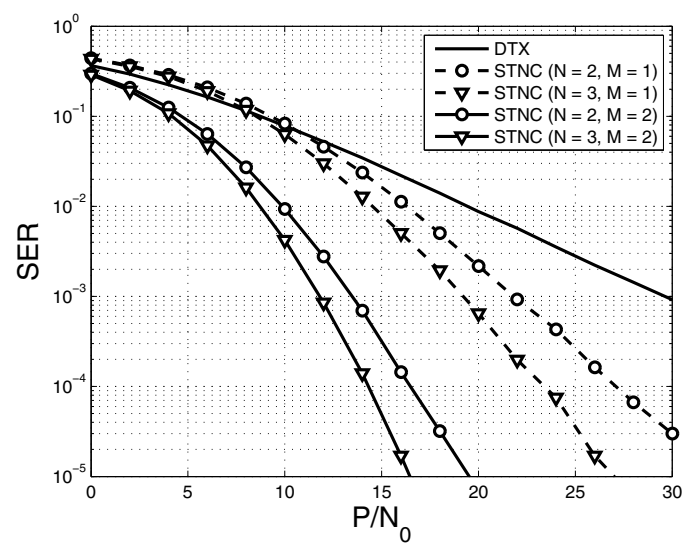

Fig. 3. SER versus SNR performance of STNC scheme for different numbers of client nodes and receive antennas for QPSK modulation.

used in DTX, where a client node directly transmits to the base node without any cooperation with other nodes.

Figure 3 presents the SER versus SNR performance of the proposed STNC for various numbers of client nodes and numbers of receive antennas. The SER performance for DTX is also presented for a comparison. For $N=2, \theta_{r}=e^{j \frac{4 r-3}{4} \pi}$ with $r=1,2$ and for $N=3, \theta_{r}=e^{j\left(\frac{1}{9}+\frac{2(r-1)}{3}\right) \pi}$ with $r=1,2,3$. From the figure, the proposed STNC clearly provides the expected diversity orders, i.e., that symbols $x_{n}$ is received with diversity order $M N$ for $N$ client nodes and $M$ receive antennas.

Figure 4 provides a performance comparison between the proposed STNC and DSTBC (with Alamouti code) for the case of $N=2$ and $M=1$. In the figure, $\Delta T_{s}$ is a synchronization error range, and the timing errors $\tau_{r}$ are assumed to be uniformly distributed in $\left[-\Delta T_{s} / 2, \Delta T_{s} / 2\right]$. From the figure, DSTBC scheme with perfect synchronization, i.e. $\Delta T_{s}=0 T_{s}$, provide about $1 \mathrm{~dB}$ advantage over the STNC scheme. When $\Delta T_{s}=0.25 T_{s}$, the DSTBC and the STNC offer similar performance although the DSTBC scheme starts to diverge at SNR greater than $25 \mathrm{~dB}$. The performance of the DSTBC scheme with $\Delta T_{s}=0.35 T_{s}$ is really bad with the observable error floor. Clearly, the proposed STNC is advantageous compared to the DSTBC when timing synchronization occurs.

\section{CONCLUSiOnS}

In this paper, we proposed a STNC scheme that utilizing transform-based coding to achieve spatial diversity with low transmission delay and eliminate the issue of imperfect frequency and timing synchronization. The scheme can be applied to any number of client nodes $N$ and provides a diversity order $N$ for each transmitted symbol. The PEP was analyzed and the code design criteria were derived to ensure achieving full diversity order. Simulations are conducted to verify the performance of the proposed scheme and to reveal its advantage over a distributed space-time block coding scheme under timing synchronization errors.

\section{REFERENCES}

[1] K. J. R. Liu, A. K. Sadek, W. Su, and A. Kwasinski, Cooperative Communications and Networking. Cambridge, UK: Cambridge University Press, 2008.

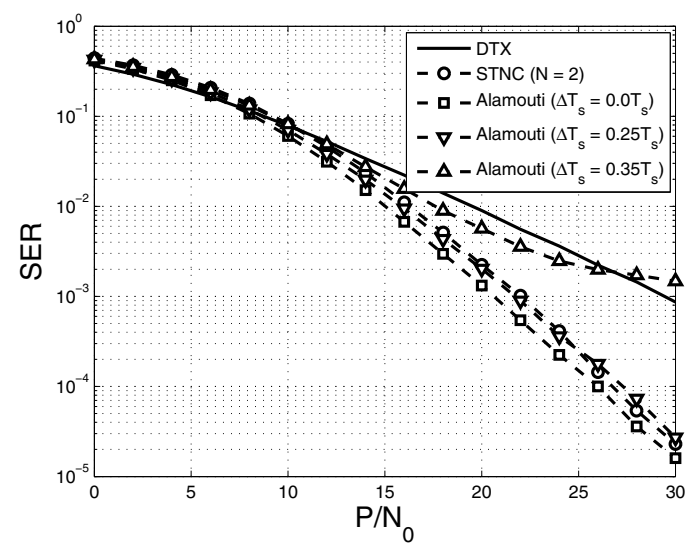

Fig. 4. SER versus SNR performance of STNC and DSTBC (Alamouti) and $M=1$ for QPSK modulation.

[2] J. N. Laneman, D. N. C. Tse, and G. W. Wornell, "Cooperative diversity in wireless networks: efficient protocols and outage behavior," IEEE Trans. Inform. Theory, vol. 50, no. 12, pp. 3062-3080, Dec. 2004.

[3] A. Sendonaris, E. Erkip, and B. Aazhang, "User cooperation diversity Part I: System description," IEEE Trans. Comm., vol. 51, pp. 1927-1938, Nov. 2003.

[4] A. K. Sadek, W. Su, and K. J. R. Liu, "Multinode cooperative communications in wireless networks," IEEE Trans. Signal Process., vol. 55, no. 1, pp. 341-355, Jan. 2007.

[5] J. N. Laneman and G. W. Wornell, "Distributed space-time coded protocols for exploiting cooperative diversity in wireless networks," IEEE Trans. Inform. Theory, vol. 49, pp. 2415-2525, Oct. 2003.

[6] V. Mudumbai, D. R. Brown, U. Madhow, and H. V. Poor, "Distributed transmit beamforming: Challenges and recent progess," IEEE Commun. Mag., vol. 47, no. 2, pp. 102-110, Feb. 2009.

[7] S. Jagannathan, H. Aghajan, and A. Goldsmith, "The effect of time synchronization errors on the performance of cooperative MISO systems," Proc. IEEE GLOBECOM, pp. 102-107, Dec. 2004.

[8] H. Q. Lai and K. J. R. Liu, "Multipoint-to-point and point-to-multipoint space-time network coding," Proc. IEEE ICASSP, Mar. 2010.

[9] X. Giraud, E. Boutillon, and J. C. Belfiore, "Algebraic tools to build modulation schemes for fading channels," IEEE Trans. Info. Theory, vol. 43, no. 3, pp. 938-952, May 1997.

[10] J. Boutros and E. Viterbo, "Signal space diversity: A power- and bandwidth-efficient diversity technique for the Rayleigh fading channel," IEEE Trans. Info. Theory, vol. 44, no. 4, pp. 1453-1467, Jul. 1998.

[11] E. Fasolo, F. Rossetto, and M. Zorzi, "Network coding meets MIMO," Proc. IEEE NetCod, pp. 1-6, Jan. 2008.

[12] "IEEE Standard for Local and Metropolitan Area Networks - Part 11: Wireless LAN Medium Access Control (MAC) and Physical Lyer (PHY) Specification," IEEE Std 802.11-2007.

[13] P. Merkey and E. C. Posner, "Optimum cyclic redundancy codes for noisy channels," IEEE Trans. Inf. Theory, vol. 30, no. 6, pp. 865-867, Nov. 1984.

[14] B. M. Hochwald and S. T. Brink, "Achieving near-capacity on a multiple-antenna channel," IEEE Trans. Commun., vol. 51, no. 3, pp. 389-399, Mar. 2003.

[15] R. A. Horn and C. R. Johnson, Topics in Matrix Analysis. New York, USA: Cambridge University Press, 2008.

[16] S. Siwamogsatham, M. P. Fitz, and J. Grimm, "A new view of performance analysis of transmit diversity schemes in correlated Rayleigh fading," IEEE Trans. Info. Theory, vol. 48, no. 4, pp. 950-956, Apr. 2002.

[17] Y. Xin, Z. Wang, and G. B. Giannakis, "Space-time diversity systems based on linear constellation precoding," IEEE Trans. Wireless Commun., vol. 2, no. 2, pp. 294-309, Mar. 2003.

[18] S. M. Alamouti, "A simple transmit diversity technique for wireless communications," IEEE J. Sel. Areas Commun., vol. 16, pp. 1451-1458, Oct. 1998.

[19] J. G. Proakis, Digital Communications. New York, USA: McGraw-Hill, 4th ed., 2001.

[20] V. Tarokh, H. Jafarkhani, and A. R. Calderbank, "Space-time block coding for wireless communications: Performance results," IEEE J. Sel. Areas Commun., no. 3, pp. 451-460, Mar. 1999. 